04

\title{
Корреляционный параметр Хейвена для диффузии фтора в супериониках $\operatorname{La}_{1-y} \mathrm{Sr}_{y} \mathrm{~F}_{3-y}$
}

\author{
(C) Н.И. Сорокин \\ Институт кристаллографии им. А.В. Шубникова ФНИЦ „Кристаллография и фотоника“ РАН, \\ Москва, Россия \\ E-mail: nsorokin1@yandex.ru
}

(Поступила в Редакцию 21 мая 2018 г.

В окончательной редакции 28 мая 2018 г.)

Процессы переноса электрического заряда (проводимость) и массы (диффузия) в супериониках $\mathrm{La}_{1-y} \mathrm{Sr}_{y} \mathrm{~F}_{3-y}$ определяются подвижными ионами фтора. Из экспериментальных данных по ионной проводимости $\sigma_{d c}$ рассчитаны коэффициенты хаотичной диффузии фтора $D_{\sigma}$ для монокристаллов $\operatorname{La}_{1-y} \mathrm{Sr}_{y} \mathrm{~F}_{3-y}$ при содержании $1,3,5,7.5,10$ и 15 mol.\% легирующего компонента $\mathrm{SrF}_{2}$. На зависимости $D_{\sigma}(y)$ наблюдается максимум в области 3-5 mol.\% $\mathrm{SrF}_{2}$. Определена величина корреляционного параметра Хейвена $H_{r}=D_{N M R} / D_{\sigma}$ $\left(D_{N M R}\right.$ - коэффициент диффузии фтора, измеренный методом магнитного резонанса на ядрах $\left.{ }^{19} \mathrm{~F}\right)$, которая характеризует механизм ионного переноса в кристаллах $\mathrm{La}_{1-y} \mathrm{Sr}_{y} \mathrm{~F}_{3-y}$. Значения $H_{r}$ составляют $0.75 \pm 0.15$, $0.45 \pm 0.15$ и $0.65 \pm 0.15$ при $400-800 \mathrm{~K}$ для 1,3 и $15-16 \mathrm{~mol} \% \mathrm{SrF}_{2}$ соответственно. Суперионик $\mathrm{La}_{0.97} \mathrm{Sr}_{0.03} \mathrm{~F}_{2.97}$ с максимальными значениями $\sigma_{d c}$ и $D_{\sigma}$, имеет минимальную величину параметра Хейвена. Полученные значения $H_{r}$ указывают, что диффузия фтора в супериониках $\mathrm{La}_{1-y} \mathrm{Sr}_{y} \mathrm{~F}_{3-y}$ не осуществляется по вакансионному механизму с участием одиночных вакансий, а происходит путем кооперативного движения ионов проводимости $\mathrm{F}^{-}$.

Работа выполнена при поддержке Федерального агентства научных организаций (соглашение № 007-ГЗ/Ч3363/26).

DOI: $10.21883 /$ FTT.2018.12.46723.102

\section{1. Введение}

Процессы образования точечных дефектов в кристаллах неорганических фторидов определяют их структурно-чувствительные свойства, в том числе перенос электрического заряда (электропроводность) и массоперенос (диффузию). Для микроскопического описания механизма ионной проводимости во фторидных супериониках, обладающих униполярной проводимостью по ионам фтора, важным является исследование параметра Хейвена (фактора корреляции) для диффузии фтора [1-5]

$$
H_{r}=D / D_{\sigma},
$$

где $D$ - экспериментально измеренный коэффициент диффузии и $D_{\sigma}-$ коэффициент диффузии, рассчитанный из измерений фтор-ионной проводимости.

Трифторид лантана является базовой матрицей для синтеза фторпроводящих суперионных проводников $\mathrm{La}_{1-y} M_{y} \mathrm{~F}_{3-y} \quad(M=\mathrm{Ca}, \mathrm{Sr}, \mathrm{Ba}) \quad[6-8] . \mathrm{LaF}_{3}$ (тисонит) имеет две структурные формы: низкотемпературная $\beta-\mathrm{LaF}_{3}$ относится к тригональной пр.гр. $P \overline{3} c 1$ („большая“ элементарная ячейка, число структурных единиц $Z=6$ ), высокотемпературная $\alpha-\mathrm{LaF}_{3}-$ к гексагональной пр.гр. $P 6_{3} / m m c$ („малая“ элементарная ячейка, $Z=2)$. Низкотемпературная модификация $\beta-\mathrm{LaF}_{3}$ является сверхструктурой по отношению к высокотемпературной $\alpha-\mathrm{LaF}_{3}$, связь между их параметрами решетки задается выражениями

$$
\begin{aligned}
& a_{\alpha}=a_{\beta} / \sqrt{3}, \\
& c_{\alpha}=c_{\beta} .
\end{aligned}
$$

Трансформация $\beta$ - $\mathrm{LaF}_{3}$ в $\alpha-\mathrm{LaF}_{3}$ происходит вследствие размытого фазового перехода при $1377 \pm 25^{\circ} \mathrm{C}$, незадолго до температуры плавления $1500 \pm 8^{\circ} \mathrm{C}$ [9].

На фазовой диаграмме системы $\mathrm{SrF}_{2}-\mathrm{LaF}_{3}$ [10] область гомогенности гетеровалентных твердых растворов $\mathrm{La}_{1-y} \mathrm{Sr}_{y} \mathrm{~F}_{3-y}$ с переменным числом атомов в элементарной ячейке составляет $0-17 \mathrm{~mol} \% \mathrm{SrF}_{2}(0 \leq y \leq 0.17$, $y$ - мольная доля $\mathrm{SrF}_{2}$ ). Кривая плавления твердых растворов имеет точку максимума при $7 \pm 1 \mathrm{~mol} \% \mathrm{SrF}_{2}$ и $1512 \pm 10^{\circ} \mathrm{C}[11]$. Введение $\mathrm{SrF}_{2}$ в матрицу $\mathrm{LaF}_{3}$ стабилизирует высокотемпературную модификацию $\alpha-\mathrm{LaF}_{3}$. Концентрационный переход от $\beta$ - к $\alpha$-форме наблюдается при 5-7 mol.\% $\mathrm{SrF}_{2}$ [12] и примерно совпадает с точкой максимума на кривой плавкости твердых растворов $\mathrm{La}_{1-y} \mathrm{Sr}_{y} \mathrm{~F}_{3-y}$.

Несоответствие ионных радиусов, электронных конфигураций и степени окисления катионов $\mathrm{La}^{3+}$ и $\mathrm{Sr}^{2+}$ приводит к ослаблению катион-анионных связей и появлению сильного структурного разупорядочения в анионной подрешетке кристаллов $\mathrm{La}_{1-y} \mathrm{Sr}_{y} \mathrm{~F}_{3-y}$

$$
\mathrm{La}^{3+} \rightarrow \mathrm{Sr}^{2+}+V_{\mathrm{F}}^{+}
$$

где $V_{\mathrm{F}}^{+}$- вакансия фтора. При охлаждении монокристаллов $\mathrm{La}_{1-y} \mathrm{Sr}_{y} \mathrm{~F}_{3-y}$, выращенных из расплава, уро- 
вень примесно-индуцированного разупорядочения анионной подрешетки сохраняется при комнатной температуре (в противоположность собственному термическому разупорядочению тисонитовой матрицы $\mathrm{LaF}_{3}$ ).

B [12-14] нами проведены измерения ионной проводимости $\sigma_{d c}$ монокристаллов $\mathrm{La}_{1-y} \mathrm{Sr}_{y} \mathrm{~F}_{3-y}$ при содержании 1, 3, 5, 7.5, 10 и 15 mol.\% компонента $\mathrm{SrF}_{2}$. Обнаружен нелинейный рост $\sigma_{d c}$ твердых растворов с изменением состава $(y)$. Кристалл $\mathrm{La}_{0.95} \mathrm{Sr}_{0.05} \mathrm{~F}_{2.95}$ $(y=0.05)$ обладает максимальной фтор-ионной проводимостью $\sigma_{d c}=3 \cdot 10^{-4} \mathrm{~S} / \mathrm{cm}$ при комнатной температуре $(293 \mathrm{~K})$, превышающей собственную проводимость матрицы $\beta-\mathrm{LaF}_{3}[12,15]$ на $\sim 4-5$ порядков.

Для исследования диффузии во фторпроводящих кристаллах классический метод радиоактивных меток не применим из-за отсутствия подходящих радиоактивных изотопов фтора. Изучение коэффициентов диффузии в супериониках, обладающих высокой подвижностью ионов фтора, может быть выполнено с применением диффузионной методики магнитного резонанса на ядрах ${ }^{19} \mathrm{~F}$ в градиентном магнитном поле (спиновое эхо) [16], которая позволяет выделить движение конкретного типа изотопа из общего диффузионного процесса. Сравнение данных по электропроводности и ${ }^{19} \mathrm{~F}$ ЯМР особенно важны для выявления природы фтор-ионного переноса в суперионных проводниках.

В работе [17] методом ${ }^{19} \mathrm{~F}$ ЯМР в импульсном градиенте магнитного поля были измерены коэффициенты диффузии фтора $D_{N M R}$ в суперионных монокристаллах $\mathrm{La}_{1-y} \mathrm{Sr}_{y} \mathrm{~F}_{3-y}$ при содержании 0.01, 0.3, 1, 3, и $16 \mathrm{~mol} \% \mathrm{SrF}_{2}$. В этой работе для трех кристаллов $\mathrm{La}_{1-y} \mathrm{Sr}_{y} \mathrm{~F}_{3-y}$ с небольшим содержанием второго компонента $0.3 ; 1$ и 3 мол.\% $\mathrm{SrF}_{2}$ приведена общая оценка величины параметра Хейвена, которая составляет $H_{r}=0.5 \pm 0.2$.

Целью работы являлось исследование корреляционного параметра Хейвена для диффузии фтора в супериониках $\mathrm{La}_{1-y} \mathrm{Sr}_{y} \mathrm{~F}_{3-y}$ при изменении концентрации от 1 до 15-16 mol.\% $\mathrm{SrF}_{2}$ в интервале температур $400-800 \mathrm{~K}$.

\section{2. Коэффициенты диффузии $D_{\sigma}$ в супериониках $\mathrm{La}_{1-y} \mathrm{Sr}_{y} \mathrm{~F}_{3-y}$, рассчитанные из измерений фтор-ионной проводимости}

Монокристаллы $\mathrm{La}_{1-y} \mathrm{Sr}_{y} \mathrm{~F}_{3-y}$ были выращены из расплава методом направленной кристаллизации Бриджмена в Институте кристаллографии РАН; описание методики роста кристаллов дано в работах $[13,18]$. Для подавления реакции пирогидролиза рост кристаллов проводили во фторирующей атмосфере, содержащей гелий и газообразные продукты пиролиза политетрафторэтилена. Химический состав монокристаллических образцов $\mathrm{La}_{1-y} \mathrm{Sr}_{y} \mathrm{~F}_{3-y}$ определялся по величине параметра элементарной ячейки [19] и соответствовал составу

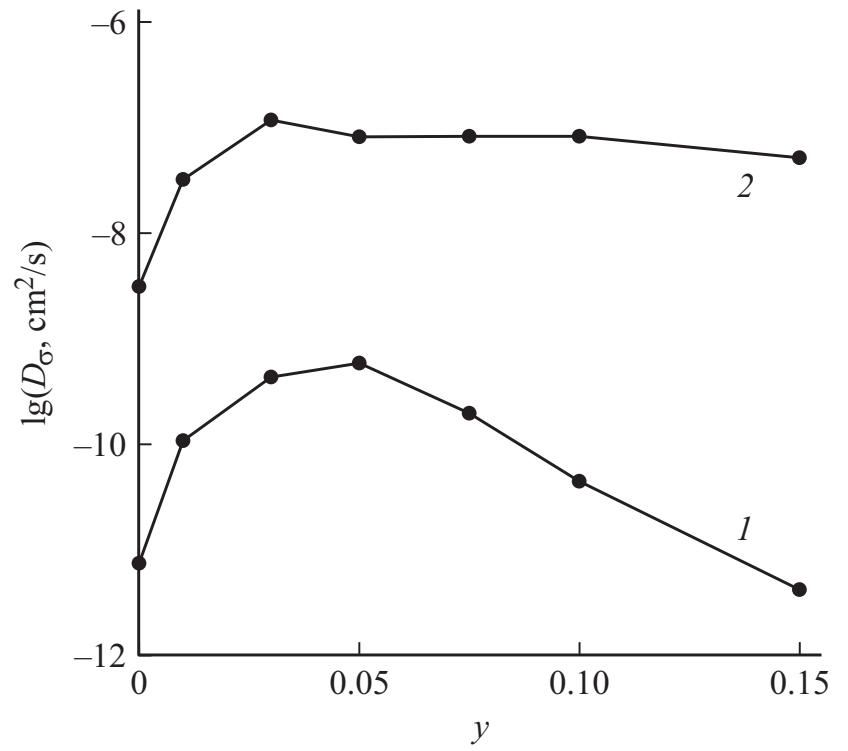

Рис. 1. Концентрационная зависимость коэффициента хаотической диффузии $D_{\sigma}(y)$ для супериоников $\mathrm{La}_{1-y} \mathrm{Sr}_{y} \mathrm{~F}_{3-y}$ при температурах 293 (1) и $500 \mathrm{~K}(2)$.

шихты с точностью \pm 1 mol.\% $\mathrm{SrF}_{2}$. Содержание примеси кислорода в кристаллах было на уровне 0.05 mass.\%.

Процессы переноса электрического заряда и диффузии в кристаллах в $\mathrm{La}_{1-y} \mathrm{Sr}_{y} \mathrm{~F}_{3-y}$ обусловлены трансляционным движением ионов фтора в анионной подрешетке. Расчет коэффициентов хаотической диффузии фтора $D_{\sigma}$ выполнен по уравнению Нернста-Эйнштейна:

$$
D_{\sigma}=\sigma_{d c} k T / n_{\mathrm{F}} q^{2}
$$

где $\sigma_{d c}-$ ионная проводимость, $n_{\mathrm{F}}-$ концентрация ионов фтора, $q$ - электрический заряд, $T$ - температуpa, $k$ - постоянная Больцмана. Данные по статической электропроводности $\sigma_{d c}$, измеренной нами переменнотоковым методом импедансной спектроскопии в интервале температур $293-800 \mathrm{~K}$ в вакууме $10^{-1} \mathrm{~Pa}$, взяты из работы [13]. Концентрация фтора в тисонитовых твердых растворах $\mathrm{La}_{1-y} \mathrm{Sr}_{y} \mathrm{~F}_{3-y}$ составляет:

$$
n_{\mathrm{F}}=2 Z(3-y) / \sqrt{3} a^{2} c .
$$

Значения параметров элементарной ячейки определены из концентрационных зависимостей [19]:

$$
\begin{aligned}
& a(y)=7.186-0.076 y, \\
& c(y)=7.352+0.094 y .
\end{aligned}
$$

На рис. 1 показана зависимость коэффициента диффузии $D_{\sigma}(y)$ кристаллов $\mathrm{La}_{1-y} \mathrm{Sr}_{y} \mathrm{~F}_{3-y}$ от состава при двух температурах 293 и $500 \mathrm{~K}$. Можно видеть, что концентрационная зависимость $D_{\sigma}(y)$ при $293 \mathrm{~K}$ имеет явно выраженный максимум в области составов 3-5 mol.\% $\mathrm{SrF}_{2}$ $(y=0.03-0.05)$. Такой же максимум наблюдался нами на концентрационной зависимости ионной проводимости 
$\sigma_{d c}(y)$ [13]. С ростом температуры зависимость $D_{\sigma}(y)$ сглаживается, что приводит к размытию максимума.

Гетеровалентные замещения катионов $\mathrm{La}^{3+}$ на $\mathrm{Sr}^{2+}$ (зарядовая неоднородность катионной подсистемы) позволяют сохранить высокотемпературное структурноразупорядоченное состояние анионной подсистемы кристаллов при их охлаждении. Согласно структурным исследованиям [12] в кристаллах $\mathrm{La}_{1-y} \mathrm{Sr}_{y} \mathrm{~F}_{3-y}$ наблюдается недозаселенность кристаллографических позиций фтора, нарастающая с ростом содержания „примесного“ компонента $\mathrm{SrF}_{2}$. Дефицит ионов фтора в кристаллографических позициях, связанный с появлением катионов пониженной валентности $\mathrm{Sr}^{2+}$, приводит к росту диффузионной подвижности фтора в кристаллах при введении до $5 \mathrm{~mol} \% \mathrm{SrF}_{2}$. При содержании в кристаллах выше $5 \mathrm{~mol} \% \mathrm{SrF}_{2}$ подвижность фтора в анионной подрешетке падает вследствие усиливающихся ион-ионных взаимодействий между ними $[13,20]$, приводящих к увеличению энтальпии активации ионного переноса.

\section{3. Параметр Хейвена для диффузии фтора в супериониках $\mathrm{La}_{1-y} \mathrm{Sr}_{y} \mathrm{~F}_{3-y}$}

Коэффициенты направленной диффузии $D_{N M R}$, измеренные методом ${ }^{19} \mathrm{~F}$ ЯМР диффузометрии (спиновое эхо) в монокристаллах супериоников $\mathrm{La}_{1-y} \mathrm{Sr}_{y} \mathrm{~F}_{3-y}$ с 1, 3 и 16 мол.\% $\mathrm{SrF}_{2}$, взяты из работы [17]. В таблице приведены рассчитанные значения параметра Хейвена $H_{r}$ для трех кристаллов $\mathrm{La}_{1-y} \mathrm{Sr}_{y} \mathrm{~F}_{3-y}$ с 1, 3 и $15-16$ мол.\% $\mathrm{SrF}_{2}$ при $500 \mathrm{~K}$. На рис. 2 показаны зависимости параметра Хейвена $H_{r}$ для этих кристаллов в интервале температур 400-800 K. Средние значения $H_{r}$ составляют $0.75 \pm 0.15,0.45 \pm 0.15$ и $0.65 \pm 0.15$ для кристаллов с $y=0.01,0.03$ и 0.15 соответственно. Значение $H_{r}=0.45 \pm 0.15$ для наиболее проводящего

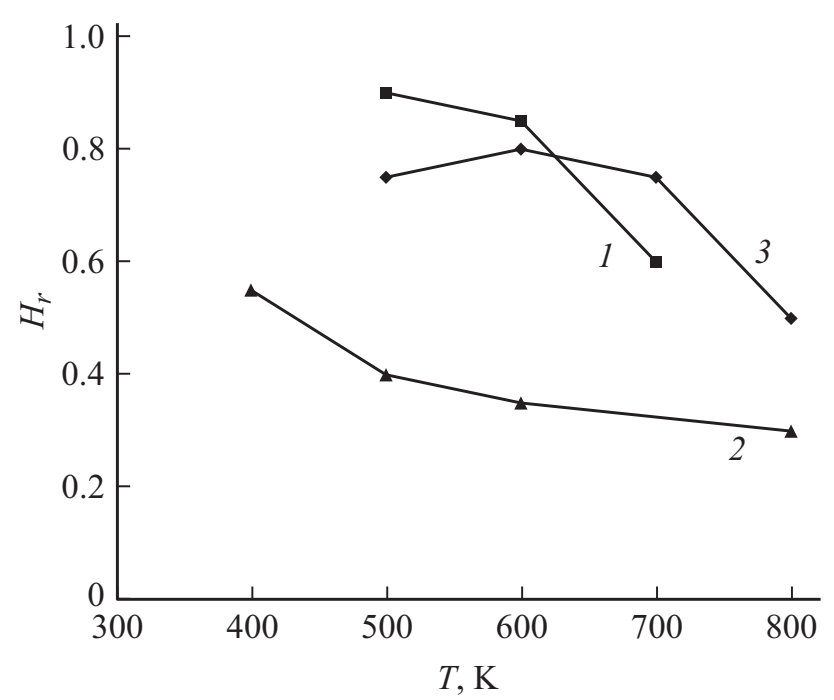

Рис. 2. Изменения параметра Хейвена $H_{r}$ при $400-800 \mathrm{~K}$ в супериониках $\mathrm{La}_{1-y} \mathrm{Sr}_{y} \mathrm{~F}_{3-y}: 1-y=0.01,2-y=0.03$ и $3-y=0.15-0.16$.
Таблица 1. Постоянно-токовая проводимость $\sigma_{d c}$, коэффициенты диффузии фтора $D_{\sigma}$ и $D_{N M R}$, параметр Хейвена $H_{r}$ в супериониках $\mathrm{La}_{1-y} \mathrm{Sr}_{y} \mathrm{~F}_{3-y}$ при $500 \mathrm{~K}$

\begin{tabular}{l|c|c|c|l}
\hline \multicolumn{1}{c|}{ Кристалл } & $\begin{array}{c}\sigma_{d c}, \\
\mathrm{~S} / \mathrm{cm}[1]\end{array}$ & $\begin{array}{c}D_{\sigma}, \\
\mathrm{cm}^{2} / \mathrm{s}\end{array}$ & $\begin{array}{c}D_{N M R}, \\
\mathrm{~cm}^{2} / \mathrm{s}[2]\end{array}$ & $H_{r}$ \\
\hline $\mathrm{La}_{0.99} \mathrm{Sr}_{0.01} \mathrm{~F}_{2.99}$ & $6.6 \cdot 10^{-3}$ & $3.3 \cdot 10^{-8}$ & $3 \cdot 10^{-8}$ & 0.9 \\
$\mathrm{La}_{0.97} \mathrm{Sr}_{0.03} \mathrm{~F}_{2.97}$ & $2.4 \cdot 10^{-2}$ & $1.2 \cdot 10^{-7}$ & $5 \cdot 10^{-8}$ & 0.4 \\
$\mathrm{La}_{1-y} \mathrm{Sr}_{y} \mathrm{~F}_{3-y}$ & $1.0 \cdot 10^{-2}$ & $5.3 \cdot 10^{-8}$ & $4 \cdot 10^{-8}$ & 0.75 \\
$(y=0.15-0.16)$ & & & &
\end{tabular}

кристалла $\mathrm{La}_{0.97} \mathrm{Sr}_{0.03} \mathrm{~F}_{2.97}$ хорошо совпадает с оценкой $H_{r}=0.35-0.4$ для кристалла $\mathrm{CaF}_{2}$ в суперионном состоянии при $1500-1700 \mathrm{~K}$, выполненной по результатам метода молекулярной динамики [21].

Полученные значения $H_{r}$ для супериоников $\mathrm{La}_{1-y} \mathrm{Sr}_{y} \mathrm{~F}_{3-y}$ указывают, что диффузия фтора не реализуется по вакансионному механизму с участием одиночных вакансий $\left(H_{r}=0.78\right.$ [22]) и свидетельствуют о кооперативном характере движения ионов проводимости $\mathrm{F}^{-}$. С ростом температуры наблюдается снижение величины $H_{r}$, что свидетельствует об усилении согласованности прыжков ионов $\mathrm{F}^{-}$. Кооперативные эффекты играют важную роль в представлениях о суперионной проводимости в кристаллах. Коллективные взаимодействия между ионами приводят к понижению свободной энергии кристалла [23] и, как отмечено в работе [24], являются причиной „,размытости“ суперионного перехода во флюоритовых кристаллах дифторидов $M \mathrm{~F}_{2}(M=\mathrm{Ca}, \mathrm{Sr}, \mathrm{Ba}, \mathrm{Cd}, \mathrm{Pb})$ [25-27] и тисонитовых кристаллах трифторидов $R \mathrm{~F}_{3}(R=\mathrm{La}, \mathrm{Ce}, \mathrm{Pr}, \mathrm{Nd})$ [9].

Величина параметра $H_{r}$ определяется координацией диффундирующего атома в кристаллах и ее можно оценить по формуле [28]:

$$
H_{r}=(1-P) /(1+P), \quad P=1 / m,
$$

где $m$ - число ближайших позиций. В анионной подрешетке тисонитовых кристаллов атомы фтора располагаются в трех типах кристаллографических позиций $\mathrm{F}_{1}$ (позиции $12 g$ пр.гр. $P \overline{3} c 1$ ), $\mathrm{F}_{2}$ (позиции $4 b$ ) и $\mathrm{F}_{3}$ (позиции $2 a$ ). В структурной модификации $\beta-\mathrm{LaF}_{3}$ позиции $\mathrm{F}_{2}$ и $\mathrm{F}_{3}$ являются структурно близкими, а в модификации $\alpha-\mathrm{LaF}_{3}$ эти позиции совпадают, образуя общую позицию $\mathrm{F}_{2,3}$. При рассмотрении динамических свойств можно рассматривать две фторные подсистемы $\mathrm{F}_{1}$ и $\mathrm{F}_{2,3}[29-31]$.

Фторы, расположенные в позициях $\mathrm{F}_{1}$ и $\mathrm{F}_{2,3}$, в разной степени участвуют в диффузионных процессах в условиях тепловой активации. По данным исследований ${ }^{19} \mathrm{~F}$ ЯМР спектроскопии и релаксометрии [29-31] при $T<T_{0} \quad\left(T_{0}=420-450 \mathrm{~K}[13]\right.$ соответствует изгибу на зависимости $\left.\sigma_{d c}(y)\right)$ наиболее вероятными являются прыжки атомов фтора в кристаллах $\mathrm{La}_{1-y} \mathrm{Sr}_{y} \mathrm{~F}_{3-y}$, в пределах системы кристаллографических позиций $\mathrm{F}_{1}$. 
При $T>T_{0}$ прыжки атомов фтора происходят в пределах всей анионной подрешетки, включающей системы кристаллографических позиций $\mathrm{F}_{1}$ и $\mathrm{F}_{2,3}$.

Для атома $\mathrm{F}_{1}$ число ближайших соседей [32] по анионной подрешетке равно $3 \mathrm{~F}_{1}+\mathrm{F}_{2,3}(m=4)$ и значение параметра Хейвена составляет $H_{r}=0.6$. Рассчитанное значение $H_{r}$ по формуле (7) для структуры тисонита удовлетворительно совпадает с полученными экспериментальными значениями $H_{r}$ для кристаллов $\mathrm{La}_{1-y} \mathrm{Sr}_{y} \mathrm{~F}_{3-y}$.

\section{4. Заключение}

Гетеровалентные замещения катионов $\mathrm{La}^{3+}$ на $\mathrm{Sr}^{2+}$ приводят к сильному структурному разупорядочению анионной подсистемы кристаллов $\mathrm{La}_{1-y} \mathrm{Sr}_{y} \mathrm{~F}_{3-y}$ с образованием вакансий фтора. Проанализированы концентрационные зависимости коэффициента хаотической диффузии фтора $D_{\sigma}$, рассчитанные из измерений фтор-ионной проводимости. Концентрационная зависимость $D_{\sigma}(y)$ имеет максимум в области составов 3-5 mol.\% $\mathrm{SrF}_{2}$. Процессы ионного транспорта и диффузии в супериониках $\mathrm{La}_{1-y} \mathrm{Sr}_{y} \mathrm{~F}_{3-y}$ определяются подвижными ионами $\mathrm{F}^{-}$. Определены значения корреляционного параметра Хейвена $H_{r}$ в супериониках $\mathrm{La}_{1-y} \mathrm{Sr}_{y} \mathrm{~F}_{3-y}$ при содержании легирующего компонента 1, 3 и 15-16 mol.\% $\mathrm{SrF}_{2}$. Полученные значения $H_{r}$ указывают на кооперативное движение ионов фтора в кристаллах $\mathrm{La}_{1-y} \mathrm{Sr}_{y} \mathrm{~F}_{3-y}$. Суперионик $\mathrm{La}_{0.97} \mathrm{Sr}_{0.03} \mathrm{~F}_{2.97}$, обладающий максимальными значениями $\sigma_{d c}$ и $D_{\sigma}$, характеризуется минимальным значением параметра Хейвена $H_{r}=0.35-0.05$ (при 400-800 K).

\section{Список литературы}

[1] K. Compaan, Y. Haven. Trans. Faraday. Soc. 52, 786 (1956).

[2] Физика электролитов / Под ред. Дж. Хладика. Мир, М. (1978). 555 c.

[3] R.E. Gordon, J.H. Strange. J. Phys. C 11, 3213 (1978).

[4] G.A. Evangelakis, V. Pontikis. Phys. Rev. B 43, 3180 (1991).

[5] V.V. Sinitsyn, O. Lips, A.F. Privalov, I.V. Murin. J. Phys. Chem. Solids 64, 1201 (2003).

[6] Н.И. Сорокин, Б.П. Соболев. Кристаллография 52, 870 (2007).

[7] Б.П. Соболев, Н.И. Сорокин. Кристаллография 59, 891 (2014).

[8] B.P. Sobolev, N.I. Sorokin, N.B. Bolotina. Photonic and Electronic Properties of Fluoride Materials / Eds. A. Tressaud, K. Poeppelmeier. Elsevier, Amsterdam (2016). 465 p.

[9] O. Greis, M.S.R. Cader. Thermochim. Acta 87, 145 (1985).

[10] B.P. Sobolev, K.B. Seiranian. J. Solid State Chem. 39, 17 (1981).

[11] Е.А. Кривандина, 3.И. Жмурова, О.И. Лямина, А.А. Быстрова, Л.П. Отрощенко, Б.П. Соболев. Кристаллография 41, 958 (1996).

[12] Н.Б. Болотина, Т.С. Черная, А.И. Калюканов, И.А. Верин, Н.И. Сорокин, Л.Е. Фыкин, Н.Н. Исакова, Б.П. Соболев. Кристаллография 60, 391 (2015).
[13] Н.И. Сорокин, Б.П. Соболев. Кристаллография 39, 889 (1994).

[14] Н.И. Сорокин, Б.П. Соболев. Электрохимия 43, 420 (2007).

[15] Н.И. Сорокин. Журн. физ. химии 76, 391 (2002).

[16] А.К. Иванов-Шиц, И.В. Мурин. Ионика твердого тела. Изд-во СПб-ГУ, СПб. (2000). Т. 1. 616 с.

[17] F. Fujara, D. Kruk, O. Lips, A.F. Privalov, V. Sinitsyn, H. Stork. Solid State Ionics 179, 2350 (2008).

[18] Н.И. Сорокин Н.И., Б.П. Соболев. ФТТ 50, 402 (2008).

[19] Б.П. Соболев, В.Б Александров, П.П. Федоров, К.Б. Сейранян, Н.Л. Ткаченко. Кристаллография 21, 96 (1976).

[20] И.В. Мурин, О.В. Глумов, Ю.В. Амелин. ЖПХ 53, 1474 (1980).

[21] А.К. Иванов-Шиц. Кристаллография 52, 131 (2007).

[22] K. Compaan, Y. Haven. Trans. Faraday. Soc. 54, 1498 (1958).

[23] Ю.Я. Гуревич, Ю.И. Харкац. Суперионные проводники. Наука, М. (1992). 288 с.

[24] Р.А. Эварестов, И.В. Мурин. ФТТ 30, 292 (1988).

[25] A.S. Dworkin, M.A. Bredig. J. Phys. Chem. 72, 1277 (1968).

[26] Р.И. Ефремова, Э.В. Матизен. Изв. СО АН СССР. Сер. хим. 2, 3 (1970).

[27] W. Shroter, J. Nolting. J. Phys. 41, 6 (1980).

[28] Дж. Маннинг. Кинетика диффузии атомов в кристаллах. Мир, М. (1971). 277 с.

[29] М.Г. Изосимова, А.И. Лившиц, В.М. Бузник, П.П. Федоров, Е.А. Кривандина, Б.П. Соболев. ФТТ 28, 2644 (1986).

[30] D. Kruk, O. Lips, P. Gumann, A.F. Privalov, F. Fujara. J. Phys.: Condens. Matter. 18, 1725 (2006).

[31] O. Lips, D. Kruk, A.F. Privalov, F. Fujara. Solid State Nucl. Magn. Res. 31, 141 (2007).

[32] А.К. Иванов-Шиц, И.В. Мурин. Ионика твердого тела. Изд-во СПб-ГУ, Том 2. СПб. (2010). 1000 с.

Редактор Т.Н. Василевская 